\title{
Trabajo colaborativo en red
}

\section{Emilio SÁEZ TORO*}

\section{Introducción}

En los aproximadamente últimos quince años hemos podido asistir a la formación y crecimiento de nuevas agrupaciones sociales existentes desde hace muy poco tiempo. Las llamadas comunidades virtuales se concretan en el funcionamiento de organizaciones formales o informales en el ciberespacio, siempre con una finalidad concreta y con una estructura social de cierta complejidad. Estas organizaciones de desarrollo virtual en su representación pero real en sus consecuencias tienen a día de hoy casi tanta diversidad en sus variantes y objetivos como las organizaciones presenciales. Sin embargo, a diferencia de las organizaciones presenciales, éstas parten con un potencial diferente en sus características que las han hecho evolucionar de diferente manera.

Es necesario tener claro que en este artículo se va a hablar de organizaciones que no tienen un correlato presencial. O sea, en los casos de los que hablamos, las organizaciones no tienen una actividad como tal que se concrete en la existencia de una sede física donde se vean los integrantes de la actividad. Además, para entender mejor la dinámica de este tipo de organizaciones hemos de imaginar que en todo caso la actividad de las mismas en cuanto a comunidades virtuales se desarrolla fundamentalmente a través de la red.

Así, en una lógica de red, podremos comprender como las prácticas colaborativas formalizadas o no, pero claras en la práctica y el ideario de estas organizaciones se convierte en una forma de hacer crecer y obtener los resultados productivos y económicos de las mismas. Pero más allá de que se pueda generar la sospecha de haber caído en cualquier determinismo tecnológico al describir la generación de nuevas formas de grupos y colaboración que se sostienen en el funcionamiento de redes telemáticas, lo que hay que considerar es el hecho de que este soporte está dando salida a elementos de organización social más propios de los tiempos de la pre-revolución industrial, ya que se establecen formas y procedimientos en los que la relación personal y directa y el trabajo artesanal (digital) son las notas predominantes. De alguna forma, encontramos en estos entornos una vuelta de tuerca que nos lleva hacia delante y al mismo tiempo hacia atrás respecto a las posibilidades y formas en las que se desarrolla.

Respecto a lo que deparará la evolución de estas formas de trabajo cooperativo en su sentido más social, evidentemente dependerá de como solidifiquen determinados modelos de funcionamiento en estos primeros años de experiencia por no decir de experimentación. No podemos perder de vista la juventud, casi infancia de estas formas de producción on line con unas características sin parangón en la historia. En todo caso, en estos momentos podemos ver algunos rasgos esenciales sobre los que se está construyendo este tipo de organizaciones y nos puede servir para comprender mejor lo que será otro plano de actividades económicas con un fuerte contenido y cooperativo y social.

\footnotetext{
* Académico del Departamento de Filosofía, Sociología y Comunicación Audiovisual y Publicidad. Universitat Jaume I. Castellón, España.
} 


\section{Actividades productivas virtuales y lógica de red}

La información es la energía y la sustancia del ciberespacio y el elemento protagonista en todos sus procesos. Sobre la acumulación informativa y su masiva circulación se ordenan los procesos de acumulación de poder y de riqueza que han hecho crecer de forma desmesurada el ámbito virtual de relación socioeconómica. Con esta información se construye y transforman los edificios simbólicos que dan cabida a la actividad humana en la red.

Por otra parte la información adquiere posibilidades de elaboración con mucho más potencial dentro de la red, ya que no sólo se trata de acumulación, sino que además las cualidades de ordenación en bases de datos, distribución masiva y múltiples formatos multimedia, interactivos, etc., la convierte en algo específico, con valor en sí mismo y objetivable en productos anteriormente inexistentes.

Así, aparecen servicios inéditos (juegos en red, servicios de información generalista personalizados, seguimientos de actividades de todo tipo, etc.) y complementos inimaginables de actividades convencionales de tipo presencial (foros de discusión sobre temas de todo tipo, venta de música y películas transmitidas a través de la red, mercadillos de segunda mano globales, etc.). Es difícil hacer una descripción exhaustiva de todas las actividades que se sustancian en un aspecto productivo en la red dada la proliferación y diversificación producida en los últimos años. Lo que ha resultado claro es que después de la fase de economía especulativa que sufrió Internet, precisamente a través de quienes menos creían en sus posibilidades, se ha llegado a un punto en el que las iniciativas que se mantienen y aparecen sí que generan un valor real no especulativo.

Muchas de estas iniciativas se fundamentan y sustancian en la colaboración entre las partes participantes como una vía, no sólo de hacer que la actividad principal se lleve mejor a término, sino como generador de valor añadido que surge de una comunicación más efectiva y la generación de metainformación ligada a esos procesos. En este sentido, las formulas cooperativas en la red, no sólo resultan más rentables en la consecución de los objetivos propuestos sino que además permiten la generación de otros productos que refuerzan la actividad.

Un ejemplo claro en este sentido es el de las tiendas on line tipo amazon.com en las que la información que generan sus usuarios en el uso de la web se convierte en una fuente de información muy rica para mejorar los procesos de compra. Los recorridos que desarrollan los clientes por los diferentes artículos configura tanto para la empresa como para el propio cliente una vía clara de mejorar el acceso a los aspectos que más interesan, con lo cual se concentran las posibilidades de compra. Así mismo, amazon aprovecha esta dinámica para crear de forma muy sencilla redes temáticas de usuarios especializados que con un mínimo esfuerzo están colaborando entre sí refinando información. De esta manera, la lógica de red hace que el tratamiento de la información digital mejore sus prestaciones en cualquier aspecto y actividad.

\section{Agrupación natural de activistas y sus actividades}

La interacción en la red permite la concentración de actividades comunicativas de personas y entidades dispersas en el espacio. Esa cualidad ha resultado tan clara que desde los primeros tiempos de Internet surgieron grupos de discusión y trabajo on line de los más variados temas. La economía incomparable que supone tener una base virtual permite desarrollar actividades que de otra manera, por el elevado coste de las 
comunicaciones convencionales (o no digitales) y su escasa flexibilidad, no sería factible.

Así, desde ocupaciones tan claramente productivas como la traducción, el diseño y la programación que se hicieron rápidamente con las ventajas que la red les ofrecía para mejorar su actividad, a actividades tan exóticas (pero no menos productivas) como el tráfico de piezas de arqueología o el de cómics históricos, así como actividades laborales tan tradicionales como la abogacía, las finanzas y banca e incluso la medicina, han encontrado en su actividad virtual una prolongación sustancial de sus actividades de siempre.

Por otra parte, no hemos de perder de vista el hecho de que han aparecido numerosas nuevas profesiones que surgen en la red y se orientan a la misma. Especialistas en usabilidad, diseñadores de flash, programadores en php, etcétera, son nombres que no existían antes del crecimiento del uso de Internet y que no tendrían sentido fuera de dicha red.

Por último, sin ser profesiones ni trabajos desde un punto de vista convencional, muchas actividades que se basan en el ocio, han conseguido generar en el agrupamiento de usuarios un cariz productivo para sus organizadores, de tal manera que estos rendimientos económicos improbables en otras dinámicas, revierten en el fortalecimiento de la estructura de agrupación de dichos usuarios y las consolidan.

Un sencillo ejemplo de este tipo de casos, puede ser una comunidad de usuarios de motocicletas, que en su página web de base contrata publicidad de casas de motos y accesorios, etcétera. Actividades como éstas no son incoherentes con los objetivos de la agrupación y por otra parte genera recursos para que la estructura técnica y humana que mantiene la agrupación continúe durante más tiempo que sin los mismos.

Hasta aquí podemos ver que la red ha permitido la agrupación de tres tipos actividades productivas ligadas al trabajo, las vinculadas a profesiones tradicionales que se adaptan a la red, las de profesiones que surgen en y para la red y las de actividades vinculadas al mantenimiento de comunidades virtuales que generan una productividad de los agrupamientos sociales que genera.

\section{La selección natural de actividades en red}

Las actividades que se realizan en el ciberespacio cubren una amplia variedad de posibilidades, aunque siempre basadas fundamentalmente en el trabajo con información (por ejemplo, trabajos de animación, diseño, programación), ya sea porque el producto desarrollado es información o porque es la parte informativa-comunicativa de un proceso diferente (actividades de gestión y comercialización, así como cualquier otra que esté relacionada con el proceso de información en la ejecución de la actividad).

Potencialmente, se pueden realizar un enorme número de actividades laborales por Internet. Además, por los procesos de control y seguimiento remoto, la variedad se multiplica. Las actividades teletrabajadas se incrementan en la medida en la que muchos procesos se están digitalizando y que la cultura del trabajo on-line se va introduciendo.

Pero hay que distinguir lo que son trabajos que se prestan a desarrollarse por Internet de una forma u otra, y lo que son actividades productivas con las características necesarias para poder sostener una comunidad íntegramente virtual. Para una empresa convencional introducir determinados procesos telemáticos, como el correo electrónico, acceso remoto a bases de datos, etcétera, supone una optimización de su funcionamiento y una mejora en el rendimiento de la misma, pero su actividad depende fundamentalmente de la parte no virtual. Sin embargo, si observamos las organizaciones 
productivas virtuales, ya sean formales o informales, encontraremos algunas características comunes vinculadas al tipo de actividad y de la información manejada en el proceso.

Entre dichas características podemos distinguir, en primer lugar como dos rasgos comunes a todas las organizaciones virtuales observadas:

- Digital o digitalizable en sus rasgos esenciales en la mayor parte de los casos. Es importante pensar en este hecho más que como cualidad consustancial, como proceso incorporado. En el caso de las comunidades virtuales en las cuales sus temas se han incorporado con la misma aparición de la red, existe una concreción de temas emergentes en información digital procesada muy dinamizada por una red de usuarios. En los casos en los que se adapta una actividad o parte de ésta, previamente existente en una faceta presencial, se introducirán en red los aspectos más estratégicos de la misma. Es significativo el hecho de que antes de que surgiesen actividades económicas exclusivamente virtuales, fue el sector industrial el que usó este tipo de tecnologías para mejorar la eficiencia de su producción a través de una mayor coordinación entre las diferentes unidades de producción, almacenaje y venta. En la raíz de la economía virtual está el toyotismo, postfordismo y el resto de ismos volcados en mejorar la coordinación de los diferentes elementos del sistema productivos con el mercado.

- Alto potencial de rendimiento por concentración espacial-virtual. La concentración de los factores productivos ha sido clave en el proceso del crecimiento económico de los últimos dos siglos. La concentración sigue siendo un factor determinante con la diferencia de que en la economía actual lo que se concentra es el proceso informativo y se mejoran los resultados en la medida en que los factores de ubicación espacial son los mejores estratégicamente para cada cometido. En ese sentido, la virtualización de la economía incrementa su valor y utilidad en la medida en la que permite actividades en la que sus participantes están más alejados físicamente, tanto más en cuanto que sin esa faceta no pudiese ser factible la actividad.

Añadidas a estas dos facetas, pero sin un carácter definitivo en los rasgos localizados en el mundo de la economía virtual, podemos encontrar:

- Alto valor añadido por conocimiento especializado. Existe una gran proporción de comunidades virtuales productivas superespecializadas. De hecho ese extremo grado de especialización junto a la cualidad de concentración virtual es una de las bazas que hace que surjan este tipo de iniciativas y que se mantengan. La participación como colaborador, usuario o cliente, trabajador, de cualquiera de estos entornos de conocimiento superespecializado supone tener un acceso al conocimiento nuclear y altamente actualizado del aspecto que se esté desarrollando.

Es muy conocido el desarrollo que tienen los sistemas informáticos de código libre, especialmente el sistema operativo Lynux que mejora en muchos aspectos a los sistemas operativos informáticos comerciales. La enorme tarea que supone desarrollar un "artefacto" tan enorme como un sistema operativo, se ha podido llevar a cabo en la medida en la que han colaborado equipos de voluntarios de todo el mundo que se han especializado en diferentes y numerosas partes que lo componen. La especialización de los participantes en estos diseños sin el problema de su localización espacial y usando el inglés como idioma estándar para superar los problemas idiomáticos ha permitido la formación de equipos de trabajo de incalculable valor.

- Bajo valor estratégico de la información pero bajo coste de acumulación y manipulación. La cantidad y variedad de información que entra y sale de la red no tiene comparación con ningún otro momento de la historia de la humanidad. Entre toda esa 
información hay muchos elementos que por si solos no aportan nada relevante o útil, pero es en su acumulación y ordenación cuando puede resultar productiva. Por ejemplo, existe todo un mercado de tratamiento estadístico de la información que genera el uso de la red: accesos a diferentes tipos de páginas web, usos de determinados servicios en red, características de los usuarios y/o de sus equipos, ecétera.

De todas maneras, nos encontramos en un momento en el que la información es una auténtica materia prima en la que según la mirada y el proceso que se le aplique se convierte en un factor productivo más. Si por ejemplo, encontramos una página web en la que alguien explica cual es su experiencia en el uso de su vehículo, objetivamente esa información no tiene mucho valor. Sin embargo, si juntamos la descripción de la experiencia de doscientas personas con un mismo modelo de vehículo, esa información supone un juicio definitivo para valorar la calidad de dicho aparato. Ese tipo de procesos de concentración está generando modelos diferentes en nuestra relación con el mercado, ya que no sólo cuenta la información que ofrecen los productores sino que se añade la del consumidor que se convierte en prosumidor para su propio servicio.

\section{Comunidades virtuales productivas}

Antes de pasar a describir los diferentes tipos, características y funcionamiento de las comunidades virtuales productivas, es necesaria una mínima acotación de que podemos considerar en este contexto una comunidad virtual. En ese sentido, nos interesa más diferenciar lo que puede ser un grupo de personas trabajando on line en determinadas circunstancias de lo que sería específicamente una CV. Para esa diferenciación sólo es necesario atender a un elemento y es el de que ese grupo de personas desarrolle su actividad total o casi totalmente interactuando en escenarios virtuales sea cual sea su tipo.

En cuanto al aspecto productivo de la organización virtual, hemos de pensar en varios tipos de actividad que directa o indirectamente se transforman en una vertiente productiva. Así podemos encontrar organizaciones virtuales que producen servicios o productos que cobran directamente a sus clientes y organizaciones que producen y distribuyen información estratégica que es incorporada de forma directa al proceso productivo. También encontramos perfiles de agrupaciones que inicialmente se forman únicamente con un ánimo lúdico y con el paso del tiempo acaban generando una productividad económica ligada al crecimiento de dichas comunidades. En este caso, las dinámicas son totalmente diferentes ya que la forma de operar de estas últimas comunidades no está orientada a la productividad aunque acabe obteniéndola, aunque resulte un elemento más utilizado para el sostenimiento de la comunidad que para un rendimiento económico en sí.

\section{Nuevas lógicas cooperativas en las comunidades virtuales}

El establecimiento de métodos de comunicación en red en las organizaciones tiende a crear una estructura más horizontal en la distribución jerárquica de las mismas. Esto en el caso de aquellas organizaciones que se trasladan completamente o parte de su estructura comunicativa a la lógica de redes, además de introducir la gestión de la información en entornos virtuales. Realmente, todo este tipo de tecnologías puede adaptarse a cualquier estructura de poder interno pero la lógica de las mismas favorece en eficacia a aquellas organizaciones que desarrollan mecanismos de comunicación (y por tanto de relación) más igualitarios. Evidentemente, no se sugiere ninguna especie de determinismo tecnológico en el que el uso de un tipo de tecnología produzca un cambio 
en las estructuras de poder de las organizaciones. Más bien, se trata de que las organizaciones hayan adoptado estas tecnologías para mejorar sus procesos y esa adopción también supone un cambio organizativo. La incorporación de TIC a los procesos productivos no implica una democratización de las organizaciones productivas, sino más bien todo lo contrario: una simplificación de sus estructuras con una concentración de poder en sus cúpulas.

Sin embargo, existe un reverso de esta moneda, facilitado a su vez por esas mismas características que en otros contextos favorecen una intensificación de la circulación y gestión productiva de la información y la comunicación. Esas cualidades de las TIC permiten que grupos dispersos en el espacio y con escasa capacidad de concentración espacial de sus actividades de interés, dirijan sus esfuerzos a la consecución de sus objetivos en entornos virtuales desligados de las limitaciones del espacio. Este tipo de organizaciones que tienen su origen desde la "no presencia", no tienen además las limitaciones de las organizaciones que dan el paso desde sus estructuras físicas y presenciales a las virtuales, ya que estas últimas se debaten en una lógica doble de gestión del poder presencial/virtual que dificulta una transparencia comunicativa entre ambas partes.

Las organizaciones virtuales de origen tienen sus propios mecanismos de gestión del poder y mantenimiento del liderazgo pero se desarrollan todas en la lógica de la red. Resulta claro que la diversidad es otro elemento que podemos encontrar en las numerosas organizaciones que han surgido y se han organizado en entornos virtuales. Pero es de especial interés la importante lógica cooperativa y colaborativa de muchas de ellas que con distintas formulas de desarrollar esa cooperación han conseguido crecer de forma sorprendente en escaso tiempo y desarrollar un gran volumen de actividad.

De alguna manera asistimos a lo que podríamos llamar la colonización del noespacio virtual. La clara diferencia sustancial es que la actividad en este contexto no tiene un límite vinculado a áreas espaciales, sino que desarrolla su potencial respecto a entornos culturales definidos por múltiples variables, siendo la principal, sin lugar, a dudas la lingüística. A diferencia de los espacios económicos basados en el territorio el espacio cultural es fluido, expansivo o constrictivo pero nunca estático. Resulta evidente que los paradigmas culturales dominantes que se trasladan de forma directa al entorno de interacción virtual están condicionados por lo que han sido los modelos económicos espaciales que han precedido al surgimiento del espacio de interacción virtual. Pero este factor condicionante evoluciona ahora con otra lógica en la que dicha plataforma cultural dominante, basada fundamentalmente en el inglés como idioma y en la cultura anglosajona en extenso, se está modulando, mezclando y adaptando a una lógica global en la que la persecución de las mejores ideas predomina y se impone a la base cultural.

Ya sea la búsqueda del negocio en extenso, la mejor información sobre manga, programación, diseño, estrategias de juego, etc., constituyen objetivos que adaptan los flujos de información provengan de donde provengan para mejorar la persecución de dichos objetivos. En esa lógica, la diversidad cultural acaba siendo una ventaja cuando aporta elementos diferenciados que enriquecen la recreación del objetivo y se establecen las pasarelas necesarias para que dificultades como el idioma dejen de serlo.

El combustible que mueve todos estos procesos es información. Lo estratégico de dichos contenidos es lo que hace incrementar el valor de las organizaciones que se desarrollan fundamentalmente en Internet. Así mismo, la creación de ese valor es el objetivo de las agrupaciones que se concentran virtualmente. El uso de la información en dichos entornos se puede concretar en múltiples fines pero siempre se puede derivar 
directa o indirectamente una utilidad económica de los mismos. De hecho muchas organizaciones virtuales con objetivos lúdicos por su éxito de convocatoria y producción "informativa" han acabado generando ingresos derivados de la misma.

La emergencia de numerosísimas organizaciones virtuales supone también una elevadísima mortandad de las mismas. Son muchas más las intentonas de formación de organizaciones virtuales que perecen sin llegar a concretarse en algo sustancial que las que lo consiguen. La relativa facilidad con la que se pueden organizar espacios virtuales de comunicación facilitan las intentonas, pero para la consecución exitosa de este tipo de organizaciones hacen falta una multiplicidad de elementos que de no hallarse hace que dichas experiencias desaparezcan con celeridad.

\section{Estructuras organizativas en las comunidades virtuales}

A grandes rasgos podemos describir algunas de las características que deben tener estas organizaciones virtuales para que permanezcan en el tiempo:

- Existe una comunidad con un ideario o unos objetivos claros, con elementos que facilitan una identidad de grupo y una estructura de poder formal o informal pero reconocida.

- La organización produce elementos útiles (información, servicios, software, diversión, etcétera) para la consecución de los objetivos de la misma.

- Existe un número mínimo (dependiendo de la actividad) de interlocutores activos.

- Hay una clara identificación de las funciones de los participantes en la organización.

- Se dispone de un conjunto de herramientas telemáticas y técnicas para la gestión de la información y la comunicación.

Estos rasgos sirven para establecer la diferenciación en el espacio virtual de lo que es una organización propia del mismo de lo que aparentemente puede serlo pero no lo es. La formalización de cara al resto de la sociedad se establece con los mismos criterios de las organizaciones presenciales, constituyéndose como empresas, asociaciones o simples agrupaciones informales. Desde este punto de vista no hay que restar importancia en este caso a las organizaciones virtuales informales ya que tanto en volumen de integrantes como de actividad es posible que sean mucho mayores que las formales. No se nos puede pasar por alto que uno de los factores de crecimiento en el uso y actividad de Internet es la proliferación de software gratuito desarrollado por comunidades informales de programadores.

\section{Formales}

Distinguir entre comunidades virtuales formales e informales supone una distinción que se concentra más en elementos secundarios, ya que el hecho de hablar de comunidad supone una estructuración interna clara y continua en el tiempo. No se ha de perder de vista que se están desarrollando formas organizativas que después de cierto tiempo en funcionamiento buscan una vía de "formalización" que encaje en sus necesidades específicas. También es claro que un gran número de organizaciones nacen como empresas y se desarrollan en esa lógica desde un primer momento. En todo caso existe un proceso de formalización progresiva en las comunidades virtuales productivas que inicialmente se forman de manera voluntarista.

\section{Informales}


Una comunidad virtual informal, al igual que una comunidad presencial informal, es la que no está bajo formas legales establecidas. No son asociaciones constituidas o empresas en cualquiera de sus formas. Aunque no existe una estadística al respecto, la percepción que se tiene recorriendo de forma sistemática Internet, es que éstas son más abundantes que las comunidades formalizadas legalmente. Este hecho no significa en ningún caso que este tipo de comunidades sean menos eficientes y eficaces que las formalizadas, pero ya sea por su naturaleza (agrupaciones centradas en aficiones, juegos, cuestiones ideológicas, culturales, etc.) o por falta de interés en complejizar más su estructura no se suele optar por la vía de la formalización legal del grupo. Finalmente podremos comprobar el buen funcionamiento de las organizaciones virtuales informales en la medida en las que cumplan sus objetivos fundacionales.

\section{Tipologías de actividades}

Aunque la impresión inicial es que las actividades que desarrollan las comunidades virtuales es enorme en su variedad, se puede realizar una ordenación de las mismas atendiendo a la forma de estructurar su organización y al tipo de información gestionada.

En principio es típico que toda comunidad virtual tenga uno o unos pocos iniciadores que además se constituyen como el núcleo de referencia en la organización y en la gestión habitual del grupo. Ya sea una organización formal o informal la existencia de ese pequeño grupo inicial es determinante en la evolución posterior del grupo. El estilo de liderazgo y de desarrollo de la comunidad virtual que plantee este grupo será el elemento que defina de forma más clara la evolución y resultados de las organizaciones. Existen grupos iniciales que están abiertos a incrementar el grupo nuclear con posteriores incorporaciones que además de asumir responsabilidades de gestión también compartan de alguna manera la propiedad de la idea que conforma el grupo.

Las comunidades virtuales basadas en empresas de usuarios con diferentes tipos de servicios, tienen la estructura más clara. Las empresas de este tipo suelen ofrecer a sus clientes un entorno de gestión de la información en el que se relacionan con ellos de diferentes formas según el tipo de actividad. Como ejemplo de este tipo de empresas podemos observar desde las que organizan subastas (tipo ebay y mercadolibre), las que crean grupos de consumidores especializados en generar información estratégica (ciao.com y en cierto modo amazon.com), publicaciones electrónicas en las que las opiniones de sus lectores se convierten en un activo más (periodistadigital), además de todas aquellas empresas que proporcionan servicios gratuitos en Internet y aprovechan la concurrencia de público en sus plataformas para gestionar distintos tipos de campañas publicitarias dirigidas a los usuarios (plataformas de chat, de alojamiento gratuito de páginas web, correo electrónico gratuito, páginas de búsquedas, etcétera).

Pero resultan especialmente interesante los casos en los que "se organiza" a los usuarios alrededor de una actividad que resulte productiva para ellos: en amazon, los compradores pueden encontrar información estratégica para mejorar sus compras y se premia hacer comentarios de los productos, especialmente de los libros. Un caso excepcional es el ciao.com, una empresa global con presencia exclusiva en Internet en la que su valor principal es el de concentrar consumidores, segmentarlos y hacerles participar de forma remunerada en la elaboración de información valiosa evaluando productos de consumo de absolutamente todas las variedades imaginables. 
El caso de Ciao es especialmente interesante porque no sólo se incentiva económicamente la producción de información valiosa por parte de los usuarios sino que además se fomenta la interacción entre los integrantes de la misma y se establece una competición interna muy animada por ver quién produce más y mejores opiniones, tanto de los productos como de las opiniones de los demás. Ciao genera un colectivo que sirve para crear información de gran valor para las empresas de marketing (Ciao en sí es una de ellas), canaliza la realización de encuestas por terceros a públicos escogidos y por otro lado, revierte parte de sus ingresos a sus usuarios. Las personas que escriben en opiniones en este entorno adoptan una posición estratégica, no sólo escribiendo numerosas opiniones (por lo general bastante extensas) sobre productos que conoce, sino que además ejerce una labor de evaluación (que ha de justificarse) de las otras opiniones, establece un grupo de autores favoritos y desarrolla una labor de creación de lazos comunitarios que refuerza su labor de autoría evaluadora y de evaluación de opiniones. Una persona bien situada en la comunidad hace que sus opiniones generen ingresos de forma constante (aunque en muy pequeñas cantidades). El hecho de que Ciao pague a sus usuarios - autores no es tan importante como el sistema creado para incentivar comunitariamente la producción de más información y diálogo respecto a la misma. Engancharse a este tipo de dinámicas porque tiene refuerzos inmediatos por la gran cantidad de respuestas iniciales, además del incremento instantáneo de ingresos (en pequeñas cantidades) por esa actividad realizada. En resumen, este tipo de comunidades supone para muchas personas la oportunidad de adquirir cierto status en una nueva comunidad e incluso el juego de competir en esa lógica basándose en una dinámica de producción y cooperación.

Lo que aporta las características de comunidad virtual a esta organización es el establecimiento de una relación entre los usuarios que se sostiene sobre una estructura formal de estatus que el sistema dota de la simbología para su adecuado y explícito reconocimiento. Este sistema comienza a utilizarse de manera generalizada en todas las plataformas para la gestión virtual de grupos. Los diseñadores de todo tipo de plataformas para la gestión de información y comunicación, explicitan en sus diseños la necesidad de incentivar la participación. Pero evidentemente, este tipo de mecanismos que exponen una jerarquía construida basada en la participación es sólo lógica en colectivos que previamente no existen o no tienen ningún tipo de organización por si mismos. En ese sentido, es lógico pensar que las organizaciones formales que ya disponen de una estructura previa claramente establecida no necesitan dotarse en su lado virtual de este tipo de mecanismos.

También es necesario considerar, que la lógica clientelista de este tipo de sitios está orientada a un diseño de negocio que se define desde una estructura empresarial que no es visible organizativamente en los entresijos del sistema. Desde este punto de vista, se diferencia con claridad lo que es la actividad como producto y la actividad de la empresa en sí, organizando a un colectivo que comparte información.

En todo caso, siempre se puede observar una pauta generalizada de premiar la participación en los entornos virtuales. Este hecho se concreta en varios aspectos de gran interés para la organización. Por una parte, se refuerza el sentimiento de pertenencia creando una dinámica de ascenso social virtual bien establecido basado generalmente en una meritocracia participativa. Por otro lado, se aportan premios objetivos a dicha participación, en unos casos más simbólicos, como el hecho de tener acceso a contenidos reservados o a determinados entornos de gestión de la comunidad, en otros meramente económicos, como en el caso de Ciao. 


\section{Formas de producción}

La producción en estos entornos se basa fundamentalmente en la generación de información de interés estratégico. Dicho interés tiene muchas dimensiones y puede ser algo meramente instrumental. La cuestión principal es que lo que hace coherente este tipo de agrupaciones es la circulación de información valiosa. Dicha información se puede instrumentalizar con las más diversas finalidades, pero es ésta la que da cuerpo a la creación de una comunidad virtual productiva.

Hemos de pensar que las comunidades virtuales de la naturaleza que sean están formadas por personas que tienen un lado presencial que es el que sostiene sus vidas. La idea es que no hay comunidad completa en la medida en la que la presencia es un elemento parcial e irregular. En ese mismo sentido, la gran mayoría de las comunidades virtuales se orientan a proporcionar información y relaciones sociales estratégicas en relación con las actividades que sus integrantes desarrollan en la vida presencial.

La paradoja de la situación es como en muchos casos la actividad virtual en este tipo de entornos dinamiza los procesos que se desarrollan en el lado presencial. Ejemplo de cómo se sustancia este tipo de información los tenemos en los casos en que profesionales que se relacionan por la red obtienen encargos, información de utilidad para llevar a cabo mejor su cometido. En el caso de las comunidades virtuales de consumo el beneficio que se traslada al lado presencial es claramente la posibilidad de realizar mejores compras, obteniendo los mejores precios por los mejores artículos.

En este escenario las prácticas de teletrabajo han constituido un elemento catalizador para la agrupación de determinados perfiles de profesionales en comunidades virtuales de carácter formal e informal. Así, profesionales como diseñadores en todos los ámbitos, traductores, programadores, etc. tienen nodos dónde buscar y encontrar tanto la información necesaria para desarrollar su trabajo como el propio trabajo en sí.

El desarrollo de las organizaciones virtuales de consumidores en las que se premia la participación directamente con dinero e indirectamente con status dentro de la misma, llega a desvirtuar los objetivos "fundamentales" de la misma. En la medida en la que el incentivo a la participación se valora en su cuantificación en cantidad y calidad de las aportaciones, se han creado dinámicas que pervierten el sistema en su finalidad real, que es crear una valoración objetiva de productos de toda índole.

Por ejemplo, en la Web Ciao, una enorme cantidad de usuarios vierten opiniones de productos que realmente no conocen, o tienen una mínima información sobre los mismos. Así mismo, como el hecho de obtener una buena valoración por el resto de usuarios incrementa tanto el estatus dentro de la comunidad, así como el dinero percibido, existe una especie de pacto no escrito por el que la gente suele valorarse de forma generalizada de forma favorable. De todo ello resulta que la información es bastante superficial, siendo que en muchos casos los análisis de mayor extensión son simples memorandums de características, en ocasiones trasladados desde el catálogo de turno o alguna comparativa existente en Internet. En estos casos, realmente se la finalidad del uso de estas comunidades para sus integrantes tendría utilidades insospechadas en un primer momento.

\section{Conclusiones}

El escenario virtual de Internet está permitiendo la creación de numerosas organizaciones exclusivamente virtuales, basadas en el intercambio de información de 
gran interés para sus participantes. En numerosas ocasiones dichas organizaciones tienen un carácter lúdico, amateur o semiprofesional, por lo que su funcionamiento interno no suele estar excesivamente compartimentado en diferentes funciones. Sin embargo, dentro de entornos virtuales de colaboración el estímulo a la competencia y a establecer rangos meritocráticos internos resulta incentivador para los integrantes de numerosos grupos. El estatus en dichos grupos se suele establecer por la cantidad y calidad de la participación y aportaciones al grupo. Es la colaboración la que marca la mejor pauta de crecimiento y "enriquecimiento" de los grupos virtuales y son los que logran una mejor calidad en dicha colaboración los que crecen y prevalecen en el tiempo. El hecho de que las organizaciones virtuales trabajen fundamentalmente con información a través de intensos vínculos comunicativos, es además coherente con los soportes técnicos que permiten plasmar de forma clara y rápida la cantidad y validez de las aportaciones de cada uno de los miembros, además de ofrecer mecanismos para la evaluación conjunta de dicha labor por todos los componentes. Estos sistemas meritocráticos están siendo los que marcan la pauta organizativa y selectiva de estos grupos virtuales, que basan su crecimiento y proliferación en una forma necesariamente colaborativa y muy transparente.

\section{Referencias bibliográficas y de Internet}

Barcelona. 1996.

GUBERN, Roman. Del bisonte a la realidad virtual: la escena y el laberinto. Anagrama.

HUERTAS, Rafael R. Sobre la realidad virtual (Virtual o no): ensayo sobre ser y comunicación en la nueva era. Mileto Ediciones. Madrid. 2002.

BRUNOLD, J., MERZ, H., WAGNER, J. Comunidades virtuales. Ediciones Deusto. Bilbao. 2002.

LEVY, Pierre. ¿Qué es lo virtual? Paidós. Barcelona. 1999.

MALDONADO, A. Lo real y lo virtual. Gedisa. Barcelona. 1994.

PÉREZ GARCÍA, A., SALINAS, J. Comunidades virtuales al servicio de los profesionales. Educación y Biblioteca. 2001.

RHEINGOLD, Howard. La comunidad virtual: una sociedad sin fronteras. Gedisa. Barcelona. 1996.

SÁNCHEZ NAVARRO, Jordi (Ed.). Realidad virtual: visiones sobre el ciberespacio.

Devir Contenidos. Barcelona. 2004.

- Web de Consumidores de Ciao. http://www.ciao.es

- Web de venta entre particulares Ebay. http://www.ebay.es

- Periodista Digital. http://www.periodistadigital.com

- Tienda on-line Amazon. http://www.amazon.com 